CORRECTION

https://doi.org/10.1038/s41586-019-0966-0

\title{
Publisher Correction: Genomic insights into the 2016-2017 cholera epidemic in Yemen
}

François-Xavier Weill, Daryl Domman, Elisabeth Njamkepo, Abdullrahman A. Almesbahi, Mona Naji, Samar Saeed Nasher, Ankur Rakesh, Abdullah M. Assiri, Naresh Chand Sharma, Samuel Kariuki, Mohammad Reza Pourshafie, Jean Rauzier, Abdinasir Abubakar, Jane Y. Carter, Joseph F. Wamala, Caroline Seguin, Christiane Bouchier, Thérèse Malliavin, Bita Bakhshi, Hayder H. N. Abulmaali, Dhirendra Kumar, Samuel M. Njoroge, Mamunur Rahman Malik, John Kiiru, Francisco J. Luquero, Andrew S. Azman,

Thandavarayan Ramamurthy, Nicholas R. Thomson \& Marie-Laure Quilici

Correction to: Nature https://doi.org/10.1038/s41586-018-0818-3, published online 02 January 2019.

In the HTML version of this Letter, the affiliations for authors Andrew S. Azman, Dhirendra Kumar and Thandavarayan Ramamurthy were inadvertently inverted; Andrew S. Azman should be associated with 'Department of Epidemiology, Johns Hopkins Bloomberg School of Public Health, Baltimore, MD, USA'; Dhirendra Kumar should be associated with both 'Maharishi Valmiki Infectious Diseases Hospital, Delhi, India' and 'Translational Health Science and Technology Institute (THSTI), Faridabad, Haryana, India'; and Thandavarayan Ramamurthy should be associated with 'Translational Health Science and Technology Institute (THSTI), Faridabad, Haryana, India.' The PDF and print versions of the Letter were correct. The original Letter has been corrected online. 